\title{
Dynamic Behaviour of DC Motor-Based Photovoltaic Pumping Systems under Searching MPPT Algorithms
}

\author{
M. A. Elgendy, B. Zahawi, D. J. Atkinson, and D. Giaouris \\ School of Electrical, Electronic, and Computer Engineering - Newcastle University \\ m.a.elgendy@ncl.ac.uk, bashar.zahawi@ncl.ac.uk,dave.atkinson@ncl.ac.uk,damian.giaouris@ncl.ac.uk
}

\begin{abstract}
The behaviour of searching maximum power point tracking (MPPT) algorithms utilizing direct duty ratio perturbation control is shown to exhibit nonlinear phenomena, including slow scale bifurcations and chaos like behaviour, when applied to a dc photovoltaic (PV) system. The performance characteristics of a dc photovoltaic water pumping system, in which the searching MPPT algorithm is applied at the frequency of the PWM signal of the converter, are examined. The nonlinear behaviour of the system is analyzed and the effects of different parameters on system performance are discussed.
\end{abstract}

Key words: PV Pumping, MPPT, Buck Converter, Chaos.

\section{INTRODUCTION}

The cost of conventional energy sources is continuously rising while the cost of PV systems is falling, increasing the potential for the use of PV energy in large scale schemes. One of the most important applications of PV energy is in water pumping. A PV pumping system comprises a PV array, a pump controller, a motor and a pump. The ac pumping system utilizing a multi-stage centrifugal pump and an induction motor is standard for PV subterranean water pumping, while the dc pumping system utilizing a single-stage centrifugal pump and a brushed dc motor, usually a permanent magnet motor, is most common for PV shallow water pumping. In the last decade, brushless dc motors have begun to replace brushed dc motors and induction motors for small scale pumping applications.

A dc motor-pump set can be connected directly to the PV generator as is the case with most commercial dc PV pumping systems. In this case, the system operates at the intersection of the I-V curve of the PV generator and the load-line (Fig. 1). This operating point may be far from the maximum power point of the PV generator. To achieve optimum matching between the PV generator and the motor-pump set, a pump controller, normally comprised of a simple dc-dc converter, is used. The duty ratio of the converter is controlled by a MPPT algorithm to maximize the power delivered to the load. The type of dc-dc converter is chosen in accordance with the voltage ratings of the motor and the array.

A comparison between the performance of directly connected systems and that of systems using the most common MPPT algorithms has recently been presented [1]. In this paper, the dynamic behaviour of dc PV pumping systems utilizing searching MPPT algorithms is examined and analyzed showing these systems to exhibit chaos-like and quasi-periodic nonlinear characteristics under normal operating conditions.

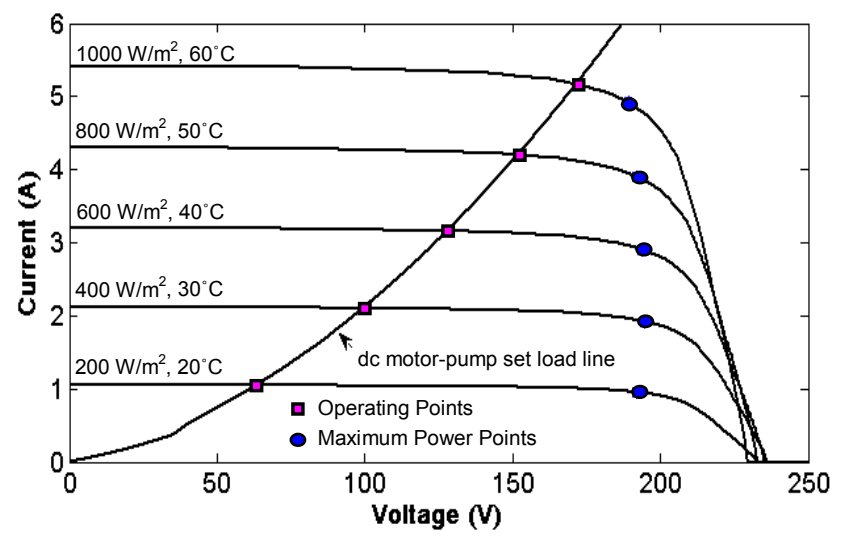

Fig. 1. Mismatch between dc motor-pump set and PV source

\section{SEARChING MAXIMUM POWER POINT TRACKING ALGORITHMS}

Searching algorithms measure the PV array output current/power or the converter output current/power and utilize the measurement to determine whether to increase or decrease a control parameter. Conventionally, the array output voltage is utilised as the control parameter and used in conjunction with a PI controller to adjust the duty ratio of the converter [2-5]. In this case, the rate of MPP tracking is much lower than the PWM rate of the converter because the system has to wait for the effects of the perturbation. More recently, a faster implementation method has been suggested where the duty ratio is used directly as the control parameter [6]. Searching MPPT algorithms do not require previous knowledge of the PV generator characteristics and do not require measurement of solar intensity and cell temperature. However, they may be affected greatly by the chosen values for the dc link capacitance of the converter, the switching frequency, and the step size of changing the control variable [1]. The most common searching algorithms used for MPP tracking are the perturb and observe (P\&O) algorithm and the incremental conductance (INC) algorithm.

In the basic form of a $\mathrm{P} \& \mathrm{O}$ algorithm [7], the operating point of the PV generator is perturbed by increasing or decreasing the converter duty ratio by a small amount and measuring the PV generator output power $\left(P_{P V}\right)$ before and after the perturbation. If the power increases, the controller continues to perturb the system in the same direction; otherwise the system is perturbed in the opposite direction. Fig. 2 shows that while the power is increasing, the direction of perturbation does not 
change. The direction of perturbation, however, reverses every PWM cycle during periods in which the power is decreasing. The operation of the $\mathrm{P} \& \mathrm{O}$ algorithm is clearly related to PV output power variations regardless of the level of PV array voltage.

During the period $\mathrm{T}=2.076-2.0813 \mathrm{sec}$, the operating point of the system is located on the negative slope $\left(d P_{P V} / d V_{P V}<0\right)$ of the array power-voltage curve (Fig. 3) hence, the correct direction of perturbation would be to increase the duty ratio of the converter thus reducing the array output voltage to move the operating point towards the MPP. When maximum power is reached at $t=2.0813 \mathrm{sec}$, the power begins to decrease, the operating point crosses the MPP and moves to the positive slope $\left(d P_{P V} / d V_{P V}>0\right)$ part of the array power-voltage curve reversing the perturbation direction. Because of the slower response time of the system compared to the speed of the algorithm, the PV output power continues to decrease even after the direction of perturbation has been reversed, confusing the $\mathrm{P} \& \mathrm{O}$ algorithm which assumes that the power reduction is a result of the new perturbation. The algorithm will therefore reverse its perturbation direction forwards and backwards until the power begins to increase at $t=2.0864$. The operating point of the system begins to move towards the MPP and crosses it again at $t=2.0911$ to the negative slope of the array powervoltage curve. The algorithm is confused again then re-corrects itself at $t=2.0963$ in the way described above.

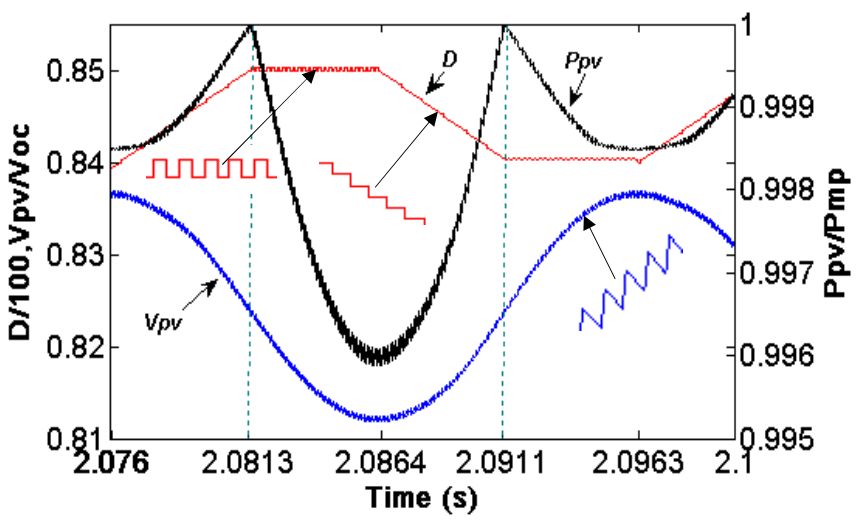

Fig. 2. Behaviour of $\mathrm{P} \& \mathrm{O}$ algorithm $\left(1000 \mathrm{~W} / \mathrm{m}^{2} \& 25^{\circ} \mathrm{C}\right)$

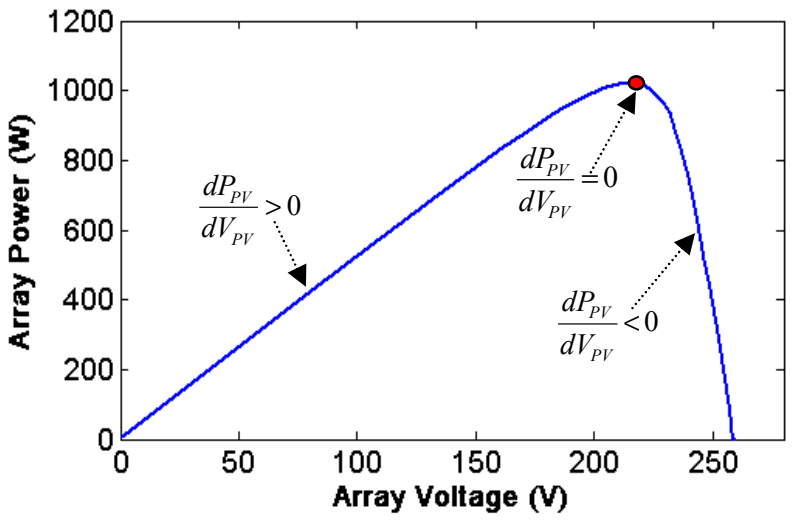

Fig. 3. Power-voltage curve of a PV generator $\left(1000 \mathrm{~W} / \mathrm{m}^{2} \& 25^{\circ} \mathrm{C}\right)$
The INC algorithm [8] is based on the fact that the P-V curve of a PV generator has only one general maximum point (Fig. 3). At this point, the derivative of the power with respect to the voltage equals zero which means that the sum of the instantaneous conductance $\left(I_{P V} / V_{P V}\right)$ and the incremental conductance $\left(d I_{P V} / d V_{P V}\right)$ equals zero (1). To the RHS and the LHS of the maximum power point, the sum of the instantaneous and incremental conductances is negative and positive, respectively $(2,3)$.

At the MPP,

$$
\frac{d I_{P V}}{d V_{P V}}=-\frac{I_{P V}}{V_{P V}}
$$

To the LHS of the MPP,

$$
\frac{d I_{P V}}{d V_{P V}}>-\frac{I_{P V}}{V_{P V}}
$$

To the RHS of the MPP,

$$
\frac{d I_{P V}}{d V_{P V}}<-\frac{I_{P V}}{V_{P V}}
$$

The incremental conductance MPPT algorithm compares the instantaneous conductance of a PV generator with its incremental conductance and decides whether to increase or to decrease the converter duty ratio accordingly. As shown in Fig. 4 , the direction of perturbation changes every crossing of the maximum power point (i.e. when the sign of $d P_{P V} / d V_{P V}$ changes). For instance, the duty ratio increases when the operating point is located on the negative slope of the array power-voltage curve (Areas $a$ and $c$ ) and decreases when it locates on the positive slope part (Areas $b$ and $d$ ).

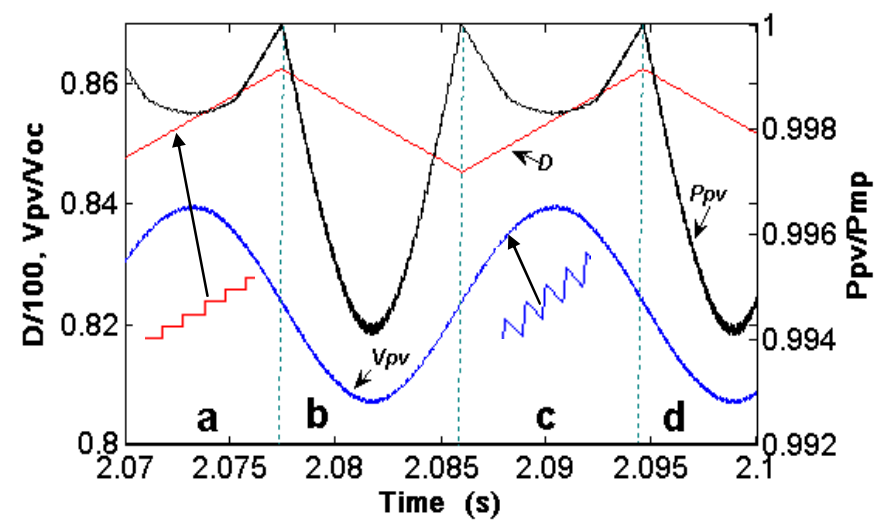

Fig. 4. Behaviour of $\mathrm{INC}$ algorithm $\left(1000 \mathrm{~W} / \mathrm{m}^{2} \& 25^{\circ} \mathrm{C}\right)$

\section{GENERAL DESCRIPTION OF THE SYSTEM}

In this paper, the nonlinear behaviour of both tracking algorithms is investigated using a dc PV pumping system comprised of $1020 \mathrm{Wp}$ PV array, a step down dc-dc converter, and a permanent magnet dc motor loaded by a centrifugal pump (Fig. 5). The system is modelled and simulated utilizing individual models of each component derived from manufactures datasheets. System operation is simulated at constant solar intensity and cell temperature at $1000 \mathrm{~W} / \mathrm{m}^{2}$ and $25^{\circ} \mathrm{C}$, respectively. The effects of the PWM frequency ( $\left.f_{P W M}\right)$, the step size $(\Delta \mathrm{D})$, and the link capacitance $\left(\mathrm{C}_{\mathrm{L}}\right)$ on system behaviour are studied. A comparison between the dynamic behaviour of the system under each MPPT algorithm is presented in the following section. 


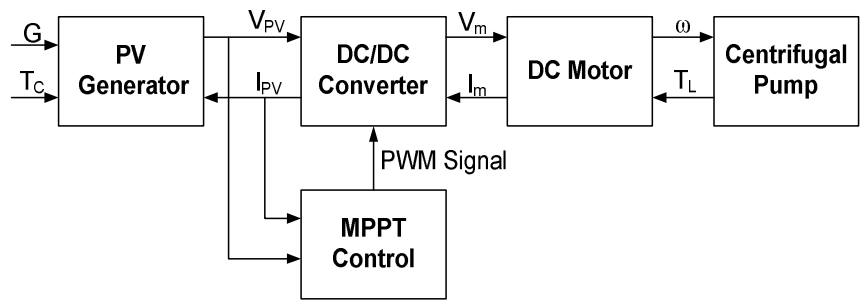

Fig. 5. DC photovoltaic pumping system block diagram

\section{Simulation RESUlts}

A PV system operating with a searching MPPT algorithm has two types of transients; one originating from variations in solar irradiance/cell temperature and the other from the perturbation of the tracking algorithm. When the system is operating under steady-state solar irradiance and temperature conditions, it is still subjected to continuous step changes in duty ratio at the PWM rate. Because of its higher speed, the direct duty ratio perturbation approach adopted in this paper gives a faster transient response and a higher tracking efficiency compared to the reference voltage perturbation approach. This, however, means that the system is never allowed to reach a steady state due to the continued perturbation of the array voltage. In this section, attention is focused on the system's response to the perturbations resulting from the operation of the tracking algorithms.

\section{A. Perturb and Observe Algorithm}

The P\&O algorithm continuously searches for the maximum power point causing the PV array voltage to fluctuate around the maximum power voltage $(213 \mathrm{~V})$ resulting in similar current and power fluctuations. As discussed above, the P\&O algorithm can not distinguish between the power variations due to the perturbation and those result from the system dynamics. Hence, the direction of perturbation may change before crossing the maximum power point producing chaos-like array voltage and current waveforms and consequently similar motor current and torque waveforms (Fig. 6). The different waveforms of the system are always bounded by two levels hence the system is globally stable. Fig. 7 shows the array voltage-motor current phase plane of the system sampled at the PWM frequency with values of dc link capacitance $(470 \mu \mathrm{F})$, PWM frequency $(10 \mathrm{KHz})$ and step size $(0.02 \%)$, chosen to give maximum power tracking efficiency [1]. The figure shows that array voltage is bounded between $208.4 \mathrm{~V}$ and $220 \mathrm{~V}$ while the motor current is bounded between $5.2 \mathrm{~A}$ and $6.25 \mathrm{~A}$. Similarly, the duty ratio of the converter changes in a chaotic pattern between $83.3 \%$ and $86.8 \%$ (Fig. 8). Similar to the duty ratio waveform shown in Fig. 2, the horizontal lines in this figure represent the power decrease periods while other lines represent the power increase. The horizontal lines do not always start after crossing the MPP which means that the P\&O algorithm may be confused any time depending on the system dynamics.

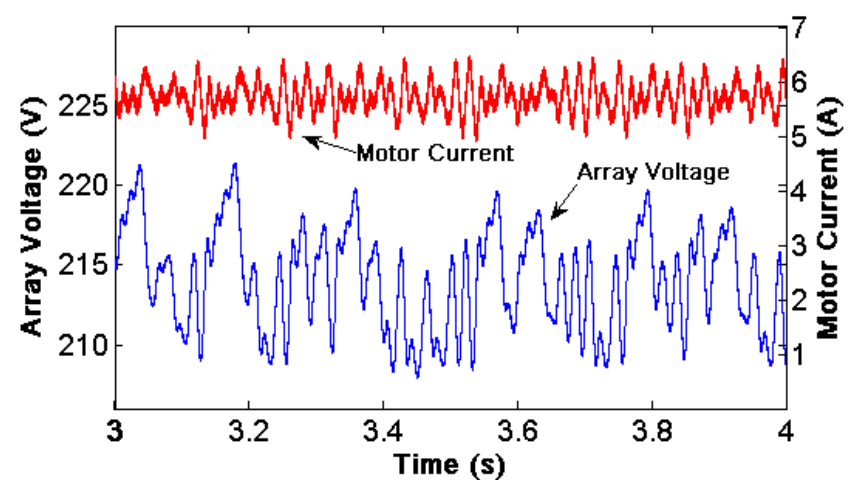

Fig. 6: Array voltage and motor current waveforms; $\mathrm{P} \& \mathrm{O}$ algorithm

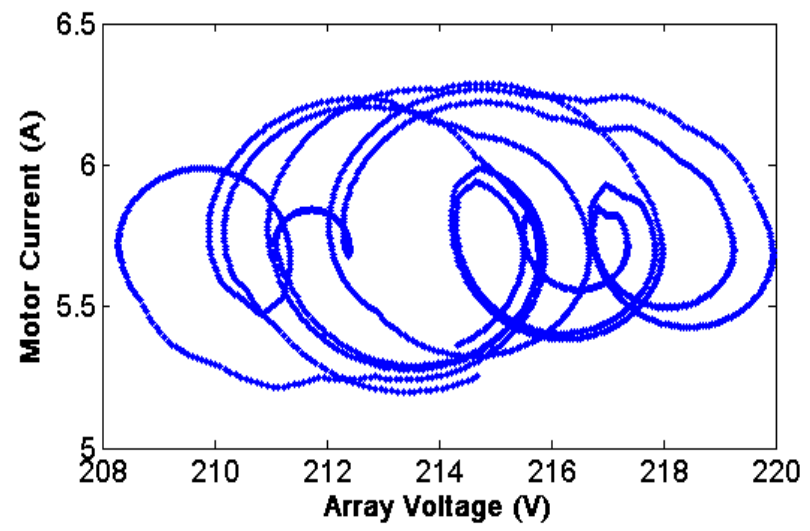

Fig. 7: Array voltage - motor current phase portrait; $\mathrm{P} \& \mathrm{O}$ algorithm

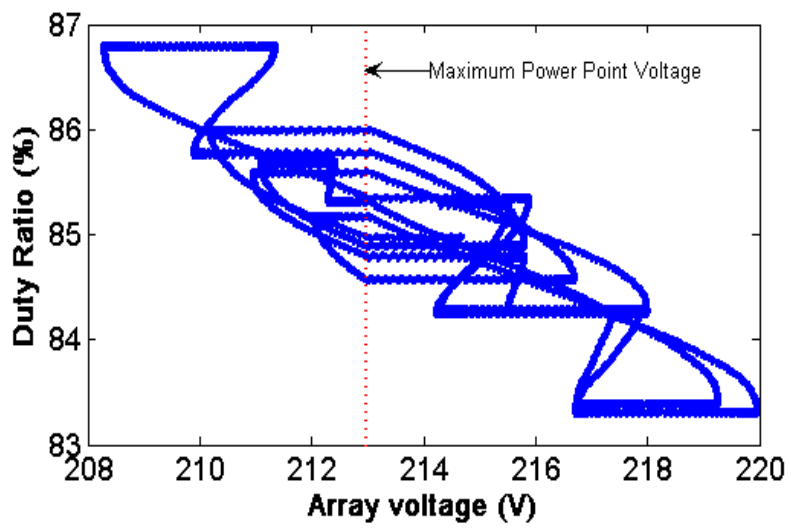

Fig. 8: Array voltage - duty ratio phase portrait; $\mathrm{P} \& \mathrm{O}$ algorithm

Fig. 9 shows the normalized frequency spectrum of the motor current. The motor current has a wide range of frequencies with maximum harmonic amplitude at $58 \mathrm{~Hz}$. The low frequency harmonics of the motor current have very low amplitude compared to the dc value hence they have little effect on motor speed. However, if the current ripple increases, the amplitudes of these low frequency components increase causing motor vibration and noise. The peak-peak motor current ripple depends on the step size, the dc link capacitance and the PWM frequency. 


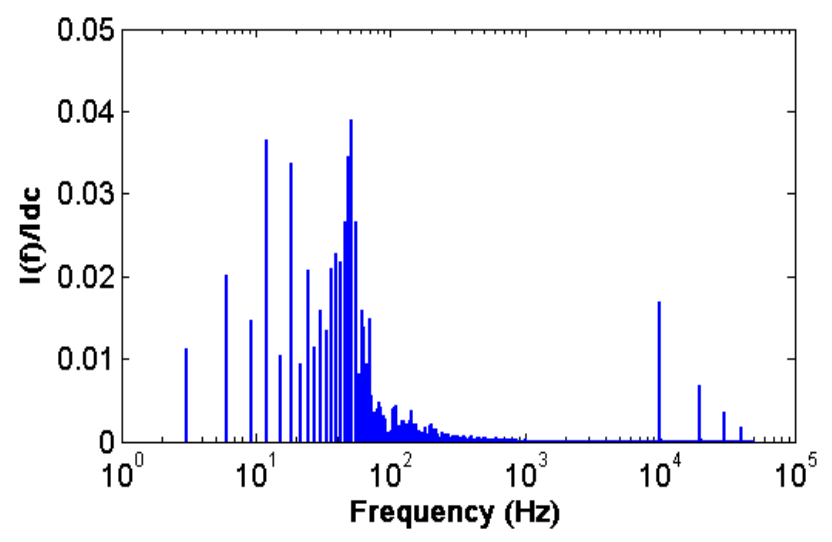

Fig. 9: Normalized frequency spectrum of motor current; P\&O algorithm

A larger step size increases the algorithm speed compared to the speed of the system response resulting in larger ripple magnitude in the array voltage and consequently in the motor current. On the other hand, a smaller step size reduces the ripple magnitude but slows down the algorithm response to radiation and temperature changes. The bifurcation diagram of the system, taking the step size as the bifurcation parameter, is shown in Fig. 10. As shown, low step sizes do not allow the system to track the maximum power. Instead, the system runs at equilibrium points near its open circuit voltage. The tracking algorithm begins to work at about $0.01 \%$ step size and works on its highest efficiency between step size values between 0.015 and 0.05 . As the step size increases, the tracking speed increases but also, the array voltage ripple increases resulting in low tracking efficiency and undesired motor operation. Occasionally, the system reaches equilibrium states where the power change with respect to the step size approaches zero. This often happens near the duty ratio limits $(0 \%$ and $100 \%)$. In this case, the duty ratio fluctuates between three levels and the power increases and decreases repeatedly with a very small amount (Fig. 11). The MPPT is lost and the system either runs as directly connected or it runs near it open circuit voltage until the irradiance and/or the temperature changes.

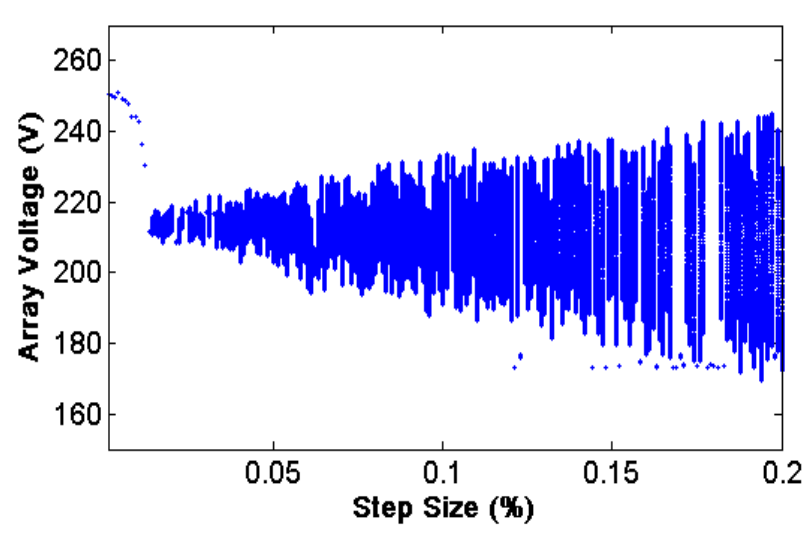

Fig. 10: Array voltage - step size bifurcation diagram; $\mathrm{P} \& \mathrm{O}$ algorithm

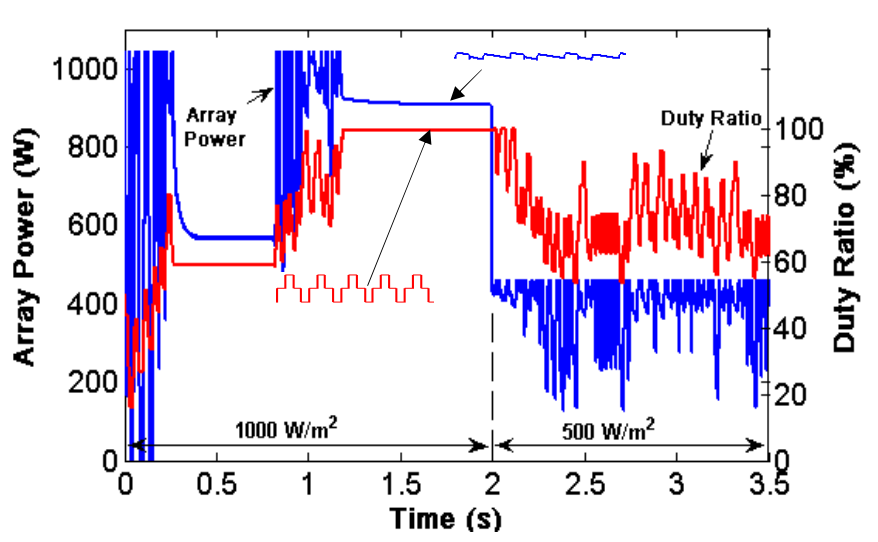

Fig. 11: Array output power and duty ratio waveforms at $0.18 \%$ step size; $P \& O$ algorithm

The bifurcation diagram of the system taking the link capacitance as the bifurcation parameter is shown in Fig. 12. A low value of link capacitance may not be enough to store the PV generated power during the off-period of the converter switch forcing the system to run at equilibrium points near its open circuit voltage. At about $100 \mu \mathrm{F}$ link capacitance, the P\&O algorithm begins to work so that the magnitude of the array voltage ripple is not affected greatly by the link capacitance. Again, MPPT may be lost at some values of the link capacitance and the system runs at equilibrium points until the irradiance and/or the temperature changes. However in this case, these equilibrium points may be closer to the maximum power point of the system.

The frequency of the converter PWM signal has a similar effect to that of the step size. Low values of PWM frequency reduce the algorithm speed resulting in slower response to radiation and temperature changes while higher PWM frequency values result in higher motor current ripple. The bifurcation diagram of the system taking the PWM frequency as the bifurcation parameter is shown in Fig. 13. At some values of the PWM frequency, MPP tracking can be lost and the system runs near its open circuit voltage.

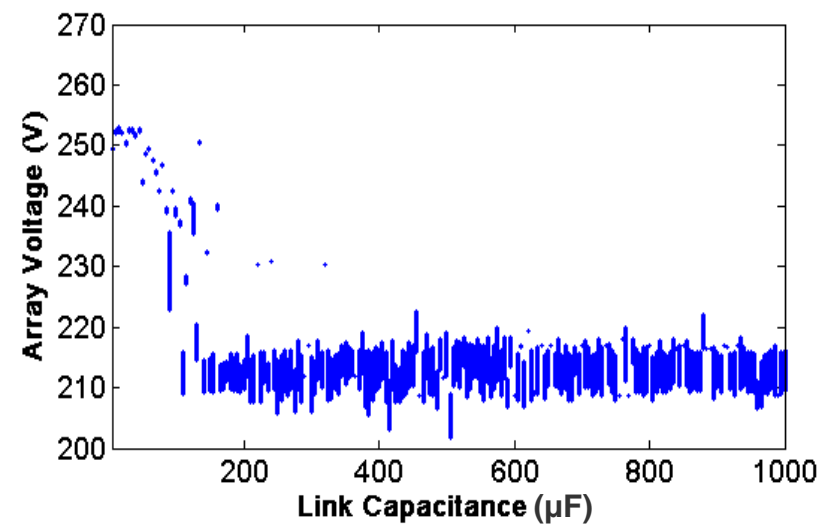

Fig. 12: Array voltage - link capacitance bifurcation diagram; $\mathrm{P} \& \mathrm{O}$ algorithm 


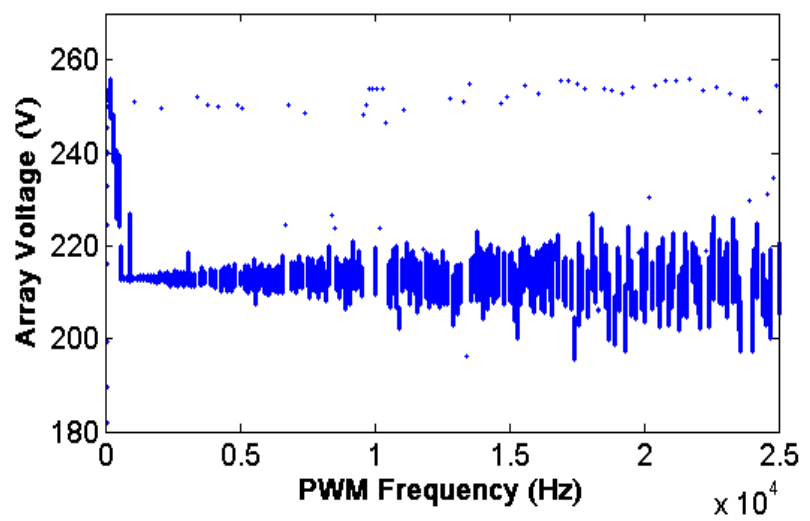

Fig. 13: Array voltage - PWM frequency bifurcation diagram; $\mathrm{P} \& \mathrm{O}$ algorithm

\section{B. Incremental Conductance Algorithm}

When using the INC algorithm, the perturbation direction changes at every crossing of the maximum power point, causing the array voltage to oscillate around the maximum power point voltage resulting in the apparently quasi-periodic voltage and current waveforms shown in Fig. 14. The different waveforms of the system are also bounded by two levels hence the system is globally stable. Fig. 15 shows the array voltagemotor current phase plane of the system sampled at the PWM frequency with values of dc link capacitance $=470 \mu \mathrm{F}, \mathrm{PWM}$ frequency $=10 \mathrm{KHz}$ and step size $=0.02 \%$, chosen to maximise array output power. As shown, the array voltage is bounded between $208.8 \mathrm{~V}$ and $217 \mathrm{~V}$ and the motor current is bounded between 4.9 A and 6.5 A. Similarly, the converter duty ratio changes in a quasi-periodic pattern between $84.5 \%$ and $86.25 \%$ (Fig. 16). This figure also illustrates how the duty ratio perturbation direction is reversed every crossing of the maximum power point $(213 \mathrm{~V})$.

The normalized frequency spectrum of the motor current is shown in Fig. 17. Unlike the $\mathrm{P} \& \mathrm{O}$ algorithm, the low frequency harmonics produced by INC algorithm are concentrated in a single tone at $58 \mathrm{~Hz}$, which has higher amplitude than those obtained using the $\mathrm{P} \& \mathrm{O}$ algorithm. If the current ripple amplitude increases, the amplitudes of the low frequency components increase and may cause motor vibration and noise. The peak current ripple again depends on the values of step size, PWM frequency, and dc link capacitance.

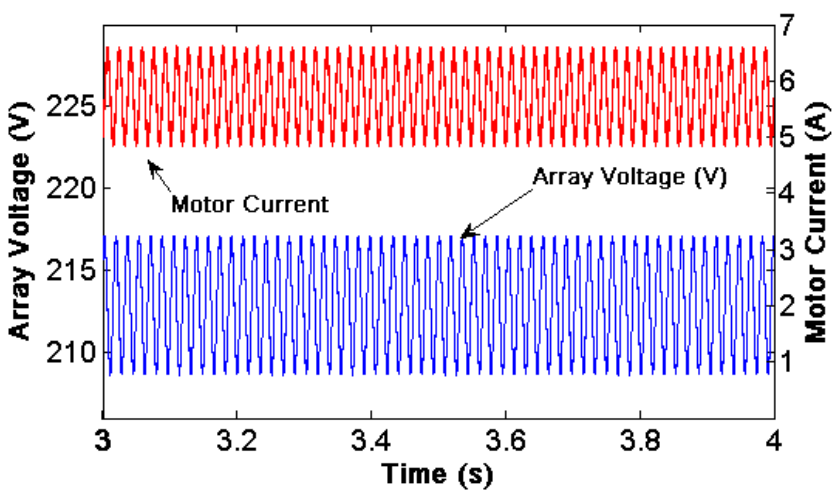

Fig. 14: Array voltage and motor current waveforms; INC algorithm

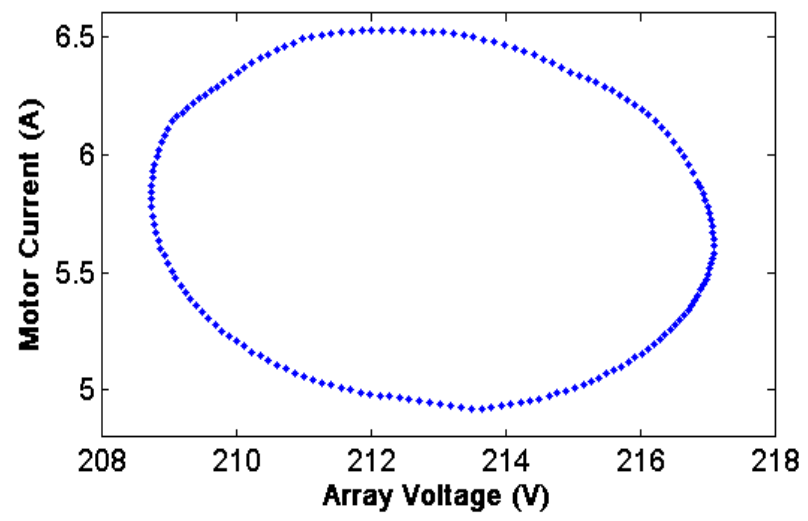

Fig. 15: Array voltage - motor current phase portrait; INC algorithm

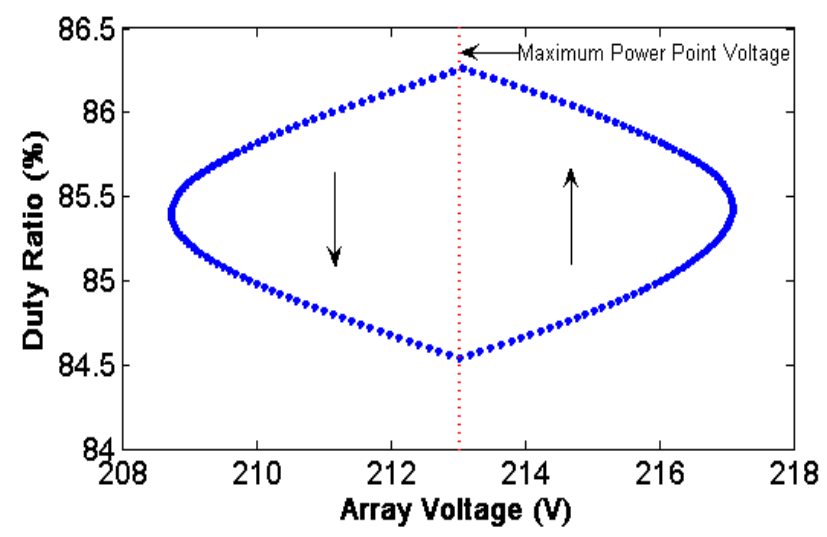

Fig. 16: Array voltage - duty ratio phase portrait; $\mathrm{P} \& \mathrm{O}$ algorithm

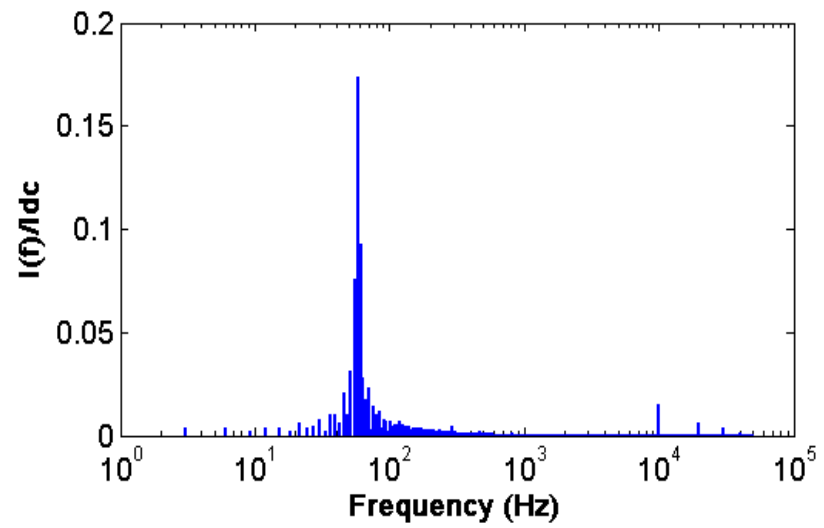

Fig. 17: Normalized frequency spectrum of motor current; INC algorithm

The bifurcation diagram of the system taking the step size as the bifurcation parameter is shown in Fig. 18. The INC algorithm begins to work at lower step size values and has smoother changes in array voltage compared to the P\&O algorithm. It works on its highest efficiency at step size values of between 0.005 and 0.05 . As the step size increases, the tracking speed increases but also, the array voltage ripple increases resulting in low tracking efficiency and undesired motor operation. 


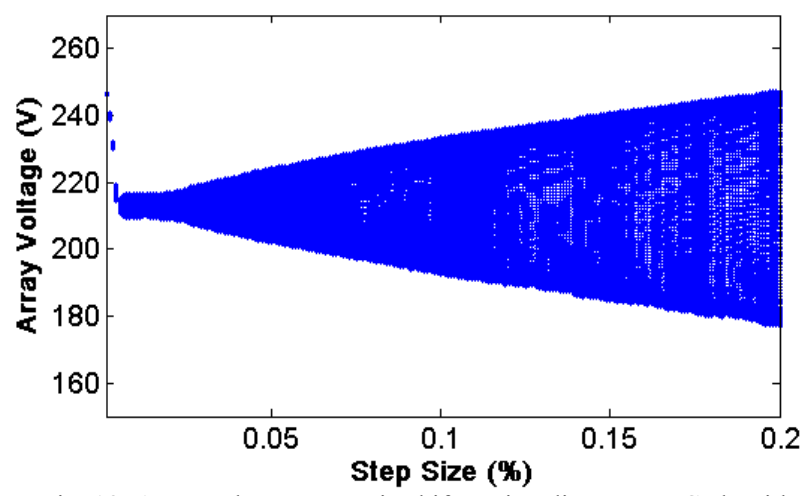

Fig. 18: Array voltage - step size bifurcation diagram; INC algorithm

The bifurcation diagram of the system taking the link capacitance as the bifurcation parameter is shown in Fig. 19. The INC algorithm begins to work at lower link capacitance values and has smoother changes in array voltage compared to the $\mathrm{P} \& \mathrm{O}$ algorithm.

Changes in PWM frequency have a similar effect to changes in step size. The bifurcation diagram of the system taking the PWM frequency as the bifurcation parameter is shown in Fig. 20. Compared with the $\mathrm{P} \& \mathrm{O}$ algorithm, the array voltage has smoother changes with the PWM frequency. Unlike the P\&O algorithm, the INC works for the whole range of PWM frequencies and system waveforms are always of the same shape.

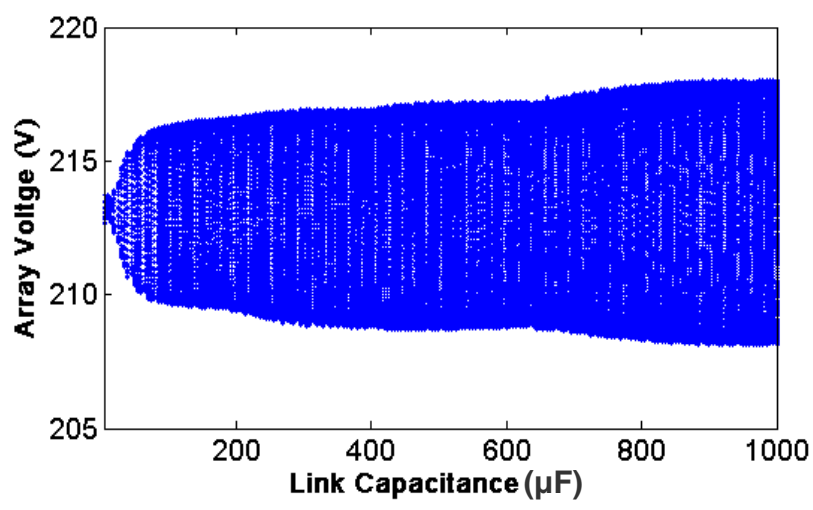

Fig. 19: Array voltage - link capacitance bifurcation diagram; INC algorithm

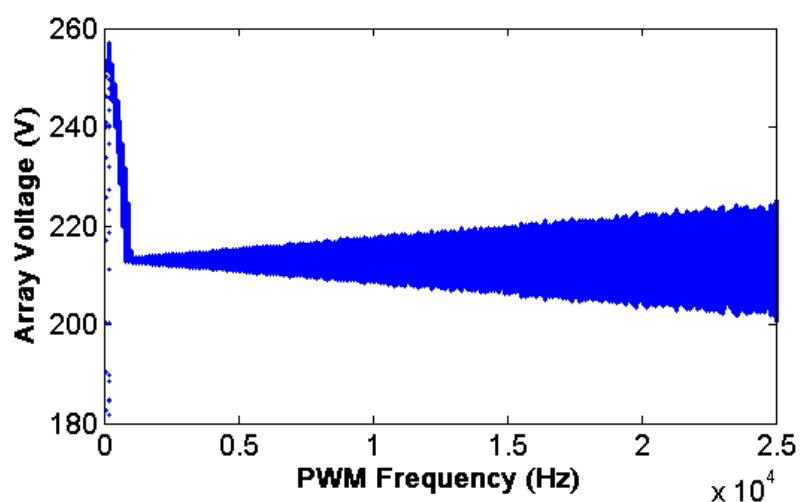

Fig. 20: Array voltage - PWM frequency bifurcation diagram; INC algorithm

\section{CONCLUSION}

An analysis of the dynamic performance of dc photovoltaic pumping systems operating with searching maximum power point tracking algorithms has been presented, showing the system to exhibit chaotic behaviour patterns when operating with $\mathrm{P} \& \mathrm{O}$ algorithms and a quasi-periodic pattern of behaviour when operating with INC algorithms. The frequency spectrum and the ripple amplitude of different current and voltage waveforms depend on the values of the PWM frequency of the MPPT converter, the value of dc link capacitance and the step size used in changing the duty ratio of the converter.

\section{ACKNOWLEDGEMENT}

The authors would like to thank Professor Soumitro Banerjee for his valuable comments and advice and would like to acknowledge the support received from the staff of the New and Renewable Energy Centre (NaREC). In addition, the first author would like to thank the Egyptian Government for sponsoring his studies.

\section{REFERENCES}

[1] M. A. Elgendy, B. Zahawi, and D. J. Atkinson., "Analysis of the Performance of DC Photovoltaic Pumping Systems with Maximum Power Point Tracking," presented at The 4th International Conference on Power Electronics, Machines and Drives 2008, York, UK, 2008.

[2] D. P. Hohm and M. E. Ropp, "Comparative study of maximum power point tracking algorithms," Progress in Photovoltaics: Research and Applications, vol. 11, pp. 47-62, 2003.

[3] V. Salas, E. Olias, A. Barrado, and A. Lazaro, "Review of the maximum power point tracking algorithms for stand-alone photovoltaic systems," Solar Energy Materials and Solar Cells, vol. 90, pp. 1555-1578, 2006.

[4] N. Femia, G. Petrone, G. Spagnuolo, and M. Vitelli, "Optimization of perturb and observe maximum power point tracking method," IEEE Transactions on Power Electronics, vol. 20, pp. 963-973, 2005.

[5] C. Hua, J. Lin, and C. Shen, "Implementation of a DSP-controlled photovoltaic system with peak power tracking," IEEE Transactions on Industrial Electronics, vol. 45, pp. 99-107, 1998.

[6] E. Koutroulis, K. Kalaitzakis, and N. C. Voulgaris, "Development of a microcontroller-based, photovoltaic maximum power point tracking control system," IEEE Transactions on Power Electronics, vol. 16, pp. 46-54, 2001.

[7] T. J. Liang, J. F. Chen, T. C. Mi, Y. C. Kuo, and C. A. Cheng, "Study and implementation of DSP-based photovoltaic energy conversion system," presented at Proceedings of the International Conference on Power Electronics and Drive Systems, 2001.

[8] K. H. Hussein, I. Muta, T. Hoshino, and M. Osakada, "Maximum photovoltaic power tracking: an algorithm for rapidly changing atmospheric conditions," IEE Proceedings: Generation, Transmission and Distribution, vol. 142, pp. 59-64, 1995. 